\title{
Testosterone antagonizes paraquat-induced cardiomyocyte senescence via the mIGF-1/SIRT1 signaling pathway
}

\author{
Xing Yu(i), Jianyi Zheng (i), Tengfei Cai 10 , Zhijian Wang (ii), and Guiping Zhu (i) \\ Cardiovascular Department, First Affiliated Hospital, Guangdong Pharmaceutical University, Guangzhou, Guangdong, China
}

\begin{abstract}
Testosterone has been demonstrated to antagonize doxorubicin-induced cardiomyocyte senescence. However, whether testosterone prevents the paraquat-induced cardiomyocyte senescence is largely unknown. The detection of SA- $\beta$-gal activity was performed using senescence $\beta$-gal staining kit and the reactive oxygen species levels were determined by reactive oxygen species assay kit. The plasmids for insulin-like growth factor 1 shRNA (sh-mIGF-1), sirtuin-1 shRNA (sh-SIRT1), scramble shRNA (sh-NC), overexpressing mIGF-1 (mIGF-1), overexpressing SIRT1 (SIRT1), and negative controls (NC) were obtained for this study. The expression of target genes was detected using quantitative real-time PCR, immunolabeling, and western blot. We found that testosterone significantly delayed the paraquat-induced HL-1 cardiomyocyte senescence as evidenced by decreasing senescence-associated $\beta$-galactosidase activity and reactive oxygen species generation, which were accompanied by the up-regulated expression of mIGF-1 and SIRT1. RNA interference to reduce mIGF-1 and SIRT1 expression showed that testosterone prevented paraquat-induced HL-1 senescence via the mIGF-1/SIRT1 signaling pathway. Furthermore, myocardial contraction was evaluated by expression of genes of the contractile proteins/enzymes, such as $\alpha$-myosin heavy chain 6 (MHC6), $\alpha$-myosin heavy chain 7 (MHC7), $\alpha$-skeletal actin (ACTA-1), and sarco/endoplasmic reticulum calcium ATPase-2 (SERCA2). Testosterone adjusted the above four gene expressions and the adjustment was blocked by mIGF-1 or SIRT1 inhibition. Our findings suggested that the mIGF-1/SIRT1 signaling pathway mediated the protective function of testosterone against the HL-1 cardiomyocyte senescence by paraquat, which provided new clues for the mechanisms underlying the antiaging role of testosterone in cardiomyocytes.
\end{abstract}

Key words: Testosterone; Paraquat; Cardiomyocyte senescence; Insulin-like growth factor-1; Sirtuin1

\section{Introduction}

With the rapid aging of the world's population, heart failure, of which the incidence increases with age, is calling for attention $(1,2)$. The heart undergoes a series of physiological and morphological changes with aging, which is called cardiac aging. Cardiac aging is a continuous and irreversible process, often accompanied by cardiomegaly, which is thought to contribute to myocardial dysfunction and failure (3). Therefore, the modulation of cardiomyocyte aging is of great significance to prevent the occurrence of heart failure.

Testosterone, the most important androgen, plays an important role in the cardiovascular system and its level gradually decreases with age in males $(4,5)$. Plasma testosterone level is inversely correlated with the incidence of multiple age-related diseases in elderly men (5). Some testosterone replacement therapies have suggested that testosterone is able to improve cognitive ability (6), enhance cardiac function (7), and delay cardiomyocyte senescence $(8,9)$. Although testosterone has multiple functions, the relationship between testosterone's effect of delaying cardiomyocyte senescence and the mIGF-1/ SIRT1 pathway has not been elucidated.

Recent studies have confirmed that insulin-like growth factor 1 (IGF-1) and sirtuin-1 (SIRT1) are associated with cardiovascular aging (10-12). IGF-1 has been proven to participate in regulation of cell signaling, senescence, and apoptosis in the heart (13). Targeted administration of IGF1 in vivo improves contractile function after experimental myocardial infarction via activated Akt (14). The attenuation of myocyte senescence by IGF-1 may delay the appearance of heart failure (15). Muscle restricted mIGF-1 has been reported to protect cardiomyocytes from toxic injury by inducing SIRT1 activity, whereas circulating other IGF-1 isoforms has no such affect (16). SIRT1 belongs to a nicotinamide adenine dinucleotide-dependent histone deacetylase, which plays a key role in regulating life and 
health $(17,18)$, as well as delays the onset of agedependent cardiac fibrosis and cell death (19). Ota et al. (20) found that testosterone inhibited oxidative stressinduced endothelial senescence via up-regulation of SIRT1.

Thus, we hypothesized that testosterone ameliorates cardiomyocyte senescence via the mIGF-1/SIRT1 signaling pathway. Firstly, we established the paraquat-induced cardiomyocyte senescence model to demonstrate whether testosterone protects cardiomyocytes from paraquatinduced senescence. Further studies elucidated that the mIGF-1/SIRT1 signaling pathway played an important role in the protective effect of testosterone against paraquatinduced cardiomyocyte senescence. Finally, we demonstrated that testosterone modified the gene expression of the contractile proteins/enzymes in senescent cardiomyocytes by mediating mIGF-1 and SIRT1 expression.

\section{Material and Methods}

\section{Cell culture}

The HL-1 mouse cardiomyocyte cell line was purchased from the Louisiana State University Medical Center (USA). Cells were cultured in Dulbecco's modified Eagle's medium (DMEM, Thermo Fisher Scientific, USA) supplemented with $5 \%$ fetal bovine serum (FBS, Life Technologies, USA), $100 \mathrm{U} / \mathrm{mL}$ penicillin (Sigma, Germany), and 100 $\mu \mathrm{g} / \mathrm{mL}$ streptomycin (Sigma) at $37^{\circ} \mathrm{C}$ in a humidified $5 \%$ $\mathrm{CO}_{2}$ incubator (371, Thermo Fisher Scientific).

\section{Testosterone treatment}

$\mathrm{HL}-1$ cardiomyocytes were exposed to $100 \mu \mathrm{M}$ paraquat for $1 \mathrm{~h}$. After trypsin digestion, the cardiomyocytes were re-seeded in plates and cultured with DMEM containing different concentrations of testosterone $(0.001$, $0.01,0.1,1 \mu \mathrm{M})$. The specific cultivation time was determined in the preliminary experiment.

\section{SA- $\beta$-galactosidase staining}

The feature of cell senescence was evaluated by enhanced senescence associated $\beta$-galactosidase (SA- $\beta$ gal) activity (21). The detection of SA- $\beta$-gal activity was performed using senescence $\beta$-gal staining kit (Beyotime, China) according to the manufacturer's instruction. Briefly, cells were rinsed twice with phosphate-buffered saline (PBS), fixed with $2 \%$ formaldehyde/0.2\% glutaraldehyde for $15 \mathrm{~min}$ at room temperature and incubated with SA- $\beta$-gal staining solution for $12 \mathrm{~h}$ at $37^{\circ} \mathrm{C}$ in humidified incubator without $\mathrm{CO}_{2}$. The cells were observed under fluorescence microscope (MF52, Mshot, China).

\section{Determination of reactive oxygen species}

Studies have shown that the specific increase of reactive oxygen species (ROS) levels is a key to the inducing and maintaining the cellular senescence process (22). The ROS levels were determined by reactive oxygen species assay kit (Beyotime) according to the manufacturer's instruction. Briefly, HL-1 cells were seeded in culture plates at a density of $1 \times 10^{5} /$ well. After attachment, cells were washed once with PBS and incubated with $10 \mu \mathrm{M}$ $\mathrm{DCFH}-\mathrm{DA}$ at $37^{\circ} \mathrm{C}$ for $20 \mathrm{~min}$. Subsequently, the excess probe was rinsed three times with PBS to ensure that only intracellular ROS would be measured. Finally, the DCF florescence distribution of 2,000 cells was detected by a BD Accuri C6 flow cytometer (BD Biosciences, USA). The results were analyzed using FlowJo data analysis software (USA).

\section{Quantitative real-time PCR analysis}

As for mRNA detection, total RNA was extracted from treated HL-1 cells using TRIzol Reagent (Qiagen, Germany) according to the instructions, and reversed transcribed to cDNA using PrimeScript ${ }^{\mathrm{TM}}$ RT reagent kit with gDNA Eraser (Takara, China). Gene expression levels were performed on an ABI Prism 7500 system (Applied Biosystems, USA) using One Step SYBR ${ }^{\circledR}$ PrimeScript ${ }^{\circledR}$ PLUS RT-PCR Kit (Takara), with each sample prepared in triplicate according to the manufacturer's recommendation. $\beta$-actin mRNA was analyzed simultaneously as a control and used for normalization of data. The relative expression of the mRNA was quantified using the $2^{-\Delta \Delta C t}$ method. The sequences of primer pairs are listed in Table 1.

\section{Plasmid construction and cell transfection}

The plasmids for mIGF-1 shRNA (sh-mIGF-1), SIRT1 shRNA (sh-SIRT1), scramble shRNA (sh-NC), overexpressing mIGF-1 (mIGF-1), overexpressing SIRT1 (SIRT1), and

Table 1. Sequences of all primer pairs in the study.

\begin{tabular}{lll}
\hline Gene name & \multicolumn{1}{c}{ Forward primer $\left(5^{\prime}-3\right)$} & \multicolumn{1}{c}{ Reverse primer $\left(5^{\prime}-3^{\prime}\right)$} \\
\hline mIGF-1 & CGGCAGGAGACATTTGATTTG & TCTTTCTCCTCTCTCCCTTCTT \\
SIRT1 & GTAAGCGGCTTGAGGGTAAT & GTTACTGCCACAGGAACTAGAG \\
$\beta$-actin & GAGGTATCCTGACCCTGAAGTA & CACACGCAGCTCATTGTAGA \\
ACTA-1 & GAGGTATCCTGACCCTGAAGTA & CACACGCAGCTCATTGTAGA \\
MHC6 & CACTTCTCCTTGGTCCACTATG & GGGAGGACTTCTGGTACAAAC \\
MHC7 & CCATCTCTGACAACGCCTATC & GGATGACCCTCTTAGTGTTGAC \\
SERCA2 & TACCTGGCTATTGGCTGTTATG & GGAAATGACTCAGCTGGTAGAA \\
\hline
\end{tabular}


Table 2. Sequence of sh-mIGF-1, sh-SIRT1, and sh-NC mRNA.

\begin{tabular}{lr}
\hline Name & shRnA sequence \\
\hline sh-mIGF-1 & ACCGGGCACCTGCAATAAAGATACACATCATACTCGAGTATGATGTGTATCTTTATTGCAGGTGCTTTTTTGAATTC \\
sh-SIRT1 & ACCGGGATGCTGTGAAGTTACTGCTACTCGAGTAGCAGTAACTTCACAGCATCTTTTTTGAATTC \\
sh-NC & ACCGGCCTAAGGTTAAGTCGCCCTCGCTGAGCGAGGGCGACTTAACCTTAGGTTTTTGAATTC \\
\hline
\end{tabular}

negative controls (NC) were obtained from Gene Denovo Biotechnology Company (China). The sequence of sh-mIGF-1, sh-SIRT1, and sh-NC are shown in Table 2. The HL-1 cells were transfected with overexpressing RNAs and shRNAs plasmids using lipid-based reagent lipofectamineTM 2000 (Invitrogen) according to the manufacturer's instruction. At $48 \mathrm{~h}$ post transfection, transfection efficiency was verified by quantitative real-time $\mathrm{PCR}$ to determine the relative expression levels of mIGF-1 or SIRT1. As shown in Supplementary Figure S1B and C, the mIGF-1 and SIRT1 overexpression vectors were successfully constructed and transfected, which were used in the study. As shown Supplementary Figure S1D and E, the efficiency of shRNAs to knock down corresponding RNA expression was tested, and the most efficient shRNAs (sh-mIGF-1 2 and sh-SIRT1 3) were used in the study.

\section{Western blot analysis}

Cells were lysed in radio immunoprecipitation assay (RIPA) buffer (Vazyme, China) supplemented with protease inhibitor cocktail (Roche, Switzerland) on ice for $15 \mathrm{~min}$, followed by centrifugation $(12000 \mathrm{~g}$ for $5 \mathrm{~min}$ at $4^{\circ} \mathrm{C}$ ) to remove cell debris. Protein concentrations were determined by BCA Protein Assay kit (Thermo Fisher Scientific) according to the manufacturer's instruction. Equal mass of total protein was separated by $10 \%$ SDS-PAGE (Vazyme), and then transferred to a polyvinylidene difluoride (PVDF) membrane (Invitrogen). The PVDF membranes were blocked with $5 \%$ skim milk (BD Biosciences) at room temperature for $1 \mathrm{~h}$, rinsed, and then incubated overnight at $4^{\circ} \mathrm{C}$ with the following primary antibodies: mIGF-I (1:1,500 dilution, Cell Signaling Technology, USA) and SIRT1 (1:1,000 dilution, Cell Signaling Technology). After removing the primary antibody, the membranes were incubated at room temperature for 30 min with horseradish peroxidase-conjugated secondary antibody (1:1000 dilution, Cell Signaling Technology). The protein blots were detected using a high signal ECL western blotting substrate (Tanon, China). Protein expression levels were quantified by densitometry using Image J software (National Institutes of Health, USA) and normalized to $\beta$-actin.

\section{Immunofluorescence assay}

At indicated times, cells were fixed with pre-cooled $4 \%$ paraformaldehyde (Sigma) for $15 \mathrm{~min}$, washed with PBS, and permeated with $0.5 \%$ Triton X-100 (Sigma) for 20 min at room temperature. After blocking with $5 \%$ bovine serum albumin (BSA, Sigma) for $30 \mathrm{~min}$, cells were incubated with the following primary antibody overnight at $4^{\circ} \mathrm{C}$ : mIGF-1 (1:500 dilution, Cell Signaling Technology) and SIRT1(1:500 dilution, Cell Signaling Technology), and then with the diluted fluorophore-conjugated secondary antibody (Abcam, USA) for $1 \mathrm{~h}$ at room temperature. The cells were stained with DAPI (Abcam) according to the manufacturer's instruction. Finally, images were captured using a fluorescence microscope with a digital camera (MF52, Mshot) and analyzed with Image-Pro Plus version 6.0 software (Media Cybernetics, USA).

\section{Statistical analysis}

Data are reported as means \pm SE. Analysis was performed using one-way ANOVA followed by Dunnett's multiple comparisons test, and $\mathrm{P}<0.05$ was considered as significant. All statistical analyses were performed using the Graphpad Prism 7.0 software.

\section{Results}

\section{Testosterone protected cardiomyocytes against PQ-} induced senescence

Firstly, we assessed the effects of paraquat (1,1dimethyl-4,4-bipyridilium dichloride, $\mathrm{PQ}, 100 \mu \mathrm{M})$ treatment for 24,48 , and $72 \mathrm{~h}$ on cell senescence in HL-1 cardiomyocytes by staining for SA- $\beta$-gal. The specific data of $P Q$-induced cell senescence in preliminary experiments is shown in Table 3. The ratio of senescent cells increased in a time-dependent manner, and was significantly increased in response to $100 \mu \mathrm{M} \mathrm{PQ}$ for $72 \mathrm{~h}$ (Supplementary Figure S1A). As a result, $\mathrm{PQ}(100 \mu \mathrm{M}, 72 \mathrm{~h})$ was chosen as the optimal conditions for the subsequent studies.

To investigate the role of testosterone on $\mathrm{PQ}$-induced HL-1 cardiomyocyte senescence, HL-1 cardiomyocytes were treated with different concentrations of testosterone $(0.001,0.01,0.1$, and $1.0 \mu \mathrm{M})$ in the presence of $P Q$ for $72 \mathrm{~h}$. The results showed that testosterone inhibited $\mathrm{PQ}$-induced $\mathrm{HL}-1$ cell senescence in a concentrationdependent manner, as evidenced by the decreasing ratio of SA- $\beta$-gal positive cells as well as ROS level (Figure $1 \mathrm{~A}$ and $B)$. Interestingly, the ratio of SA- $\beta$-gal positive cells and the ROS level in testosterone $(1.0 \mu \mathrm{M})$ plus PQ-treated HL-1 cells were significantly decreased compared with those treated with $P Q$ alone, but there was no difference with the 
Table 3. Comparison of cell senescence rates of the control (CK) and paraquat (PQ) groups.

\begin{tabular}{lclcc}
\hline Group & $\begin{array}{c}\text { Senescence rates } \\
\text { (means } \pm \text { SE) }\end{array}$ & Group & $\begin{array}{c}\text { Senescence rates } \\
\text { (means } \pm \text { SE) }\end{array}$ & $\begin{array}{c}\text { P value } \\
\text { (CK vs PQ) }\end{array}$ \\
\hline CK-24h & $9.09 \pm 1.60$ & PQ-24h & $12.41 \pm 0.79$ & 0.119 \\
CK-48h & $17.29 \pm 1.30$ & PQ-48h & $19.05 \pm 1.70$ & 0.584 \\
CK-72h & $26.67 \pm 1.60$ & PQ-72h & $30.88 \pm 1.11$ & 0.008 \\
\hline
\end{tabular}

Each group of cells was counted in 4 fields randomly selected under the microscope. Cell senescence rate (\%): Number of senescent cells/total number of cells $\times 100 \%$. Student's $t$-test.

untreated cells. Therefore, testosterone $(1.0 \mu \mathrm{M})$ was chosen as the optimal concentration for the subsequent studies.

\section{Testosterone promoted protein expression of mIGF-1 and SIRT1 in PQ-treated cardiomyocytes}

Previous studies have demonstrated that locally acting mIGF-1 and SIRT1 play a role in the regulation of organism health-span $(12,23)$. Thus, we further investigated whether testosterone affects the expression of mIGF-1 and SIRT1 in PQ-treated HL-1 cardiomyocytes. Firstly, we used western blot assay to detect the protein levels of mIGF-1 and SIRT1 in HL-1 cardiomyocytes with or without testosterone (Figure $1 \mathrm{C}$ ). Compared with the non-treatment groups, the expression of mIGF-1 and SIRT1 decreased significantly in PQ-treatment groups, and increased significantly in $P Q$ plus testosterone treatment groups. Moreover, immunofluorescence assay was performed to point out the localization of SIRT1 in HL-1 cardiomyocytes (Figure 1D). The data revealed that SIRT1 (red) was mostly located inside the nuclei (blue). Paraquat significantly decreased the expression of SIRT1 in HL-1 cardiomyocytes, as the mean fluorescent density reduced, while testosterone plus $P Q$ treatment significantly increased the expression. Taken together, these results suggested that testosterone promoted the expression of mIGF-1 and SIRT1 in cardiomyocytes.

\section{Testosterone inhibited PQ-induced senescence via activating mIGF-1 expression}

To further investigate whether the protective effect of testosterone against PQ-induced senescence was associated with mIGF-1 expression level, we first used western blot assay to detect the protein level of mIGF-1 in HL-1 cardiomyocytes transfected with mIGF-1 and sh-mIGF-1 (Figure 2A). Under $P Q$ plus testosterone condition, the protein expression of mIGF-1 in $\mathrm{HL}-1$ cardiomyocytes transfected with mIGF-1 was significantly increased compared to that in no transfection, and significantly reduced in HL-1 cardiomyocytes transfected with si-mIGF-1. To further validate the role of $\mathrm{mIGF}-1$ in testosterone-delayed cell senescence, the SA- $\beta$-gal assay and ROS level were assessed under the overexpression and inhibition of mIGF1condition induced by mIGF-1 or si-mIGF-1 (Figure 2B). The results showed that SA- $\beta$-gal positive cell and ROS levels were significantly decreased in $\mathrm{HL}-1$ cardiomyocytes transfected with mIGF-1 compared to that in no transfection, while significantly increased in cardiomyocytes transfected with si-mIGF-1. Taken together, these data indicated that testosterone protected HL-1 cardiomyocytes against $P Q$-induced senescence via up-regulating mIGF-1 expression.

\section{Testosterone mediated SIRT1 activity to protect HL-1 cardiomyocytes against $P Q$-induced senescence}

To explore the underlying mechanism by which testosterone activates mIGF-1 in PQ-induced HL-1 cardiomyocytes, we first evaluated the effect of mIGF-1 overexpression or inhibition on SIRT1 expression in $\mathrm{PQ}$ induced HL-1 cardiomyocytes treated with testosterone. We used western blot assay to detect the protein levels of SIRT1 in HL-1 cardiomyocytes transfected with mIGF-1 and sh-mIGF-1. The results showed that the expression of SIRT1 significantly increased in the testosterone plus mlGF-1 group compared to that in the testosterone group, and significantly decreased in the testosterone plus sh-mIGF-1 group (Figure 2A). In addition, analysis of the location and expression levels of SIRT1 were measured by immunofluorescence assay, and the results revealed that SIRT1 (red) was located inside the nuclei (blue), and the expression levels of SIRT1 in each group were consistent with western blot analysis (Figure $2 \mathrm{C}$ ). The above data indicated that the up-regulation of SIRT1 expression was regulated by $\mathrm{mIGF}-1$.

Since SIRT1 changes are in line with mIGF-1 activation, we assessed whether SIRT1 played roles in testosteronedelayed cardiomyocyte senescence by silencing or overexpressing SIRT1. We first measured the transfection efficiency by immunofluorescence assay and western blotting (Figure 2D and E). The results showed that SIRT1 transfection significantly increased the SIRT1 expression in the presence of $P Q$ plus testosterone, but sh-SIRT1 transfection significantly decreased the SIRT1 expression. Moreover, under PQ plus testosterone condition, SIRT1 overexpression accelerated the protective effect of testosterone on PQ-induced HL-1 cardiomyocyte senescence, while silencing SIRT1 triggered a significant increase in SA- $\beta$-gal positive cell and ROS levels compared to the control group (Figure 2F). In summary, these data demonstrated 

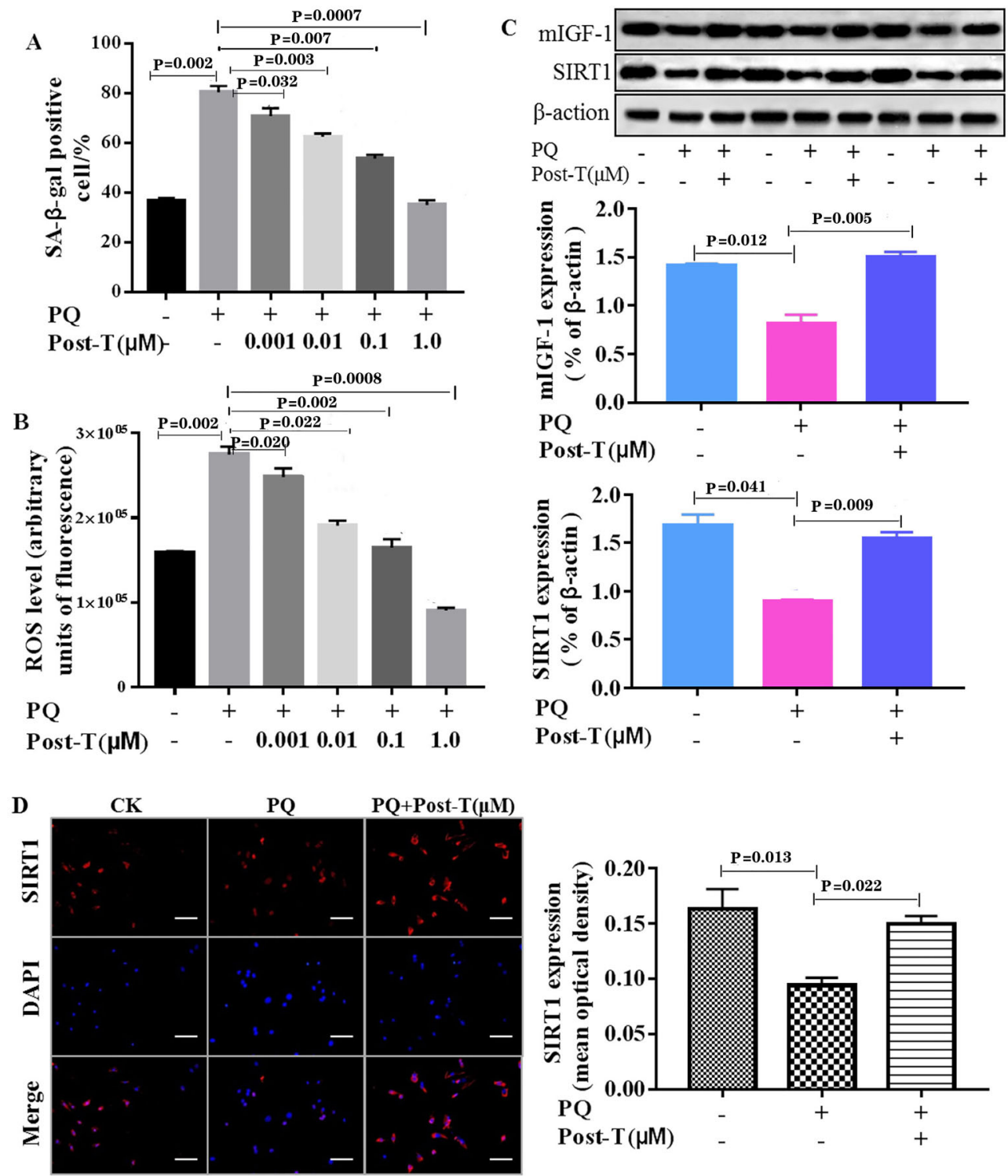

Figure 1. Testosterone $(T)$ prevents paraquat $(P Q)$-induced cell senescence in HL-1 cardiomyocytes. A, Percentages of senescenceassociated $\beta$-galactosidase (SA- $\beta$-gal)-positive $\mathrm{HL}-1$ cells after no treatment or exposure to $\mathrm{PQ}$ for $1 \mathrm{~h}$, and with or without incubation with testosterone for $72 \mathrm{~h}$ at the indicated concentrations. B, Effect of testosterone on reactive oxygen species (ROS) production of HL-1 cardiomyocytes after the same treatments as in panel A. C. Protein expression of mIGF-1 and SIRT1 in HL-1 cardiomyocytes was measured by western blotting after no treatment or exposure to $\mathrm{PQ}$ for $1 \mathrm{~h}$ post-incubated with or without testosterone $(1.0 \mu \mathrm{M})$ for $72 \mathrm{~h}$. D, Detection of SIRT1 expression in HL-1 cardiomyocytes by immunofluorescence after the same treatment as in panel C. Scale bar indicates $50 \mu \mathrm{m}$. Data are reported as means \pm SE of four independent experiments (ANOVA and Dunnett's multiple comparisons test).

that testosterone regulated the activity of SIRT1 to protect HL-1 cardiomyocytes from PQ-induced senescence.

Testosterone-activated mIGF-1/SIRT1 pathwayregulated gene expression of contractile proteins/ enzymes in cardiomyocyte senescence

Cardiomyocyte aging leads to the increase of myocardial stiffness and the decrease of systolic functions, which are related to gene expression of the contractile proteins/enzymes, including $\alpha$-myosin heavy chain 6 (MHC6) conversion to $\alpha$-myosin heavy chain 7 (MHC7), upregulating $\alpha$-skeletal actin (ACTA-1), and downregulating sarco/endoplasmic reticulum calcium ATPase-2 (SERCA2) $(16,24)$. As shown in Figure 3, PQ significantly increased the expression of ACTA1 and $\mathrm{MHC} 7$ and decreased the expression of SERCA2 and MHC6 in HL-1 cardiomyocytes, 

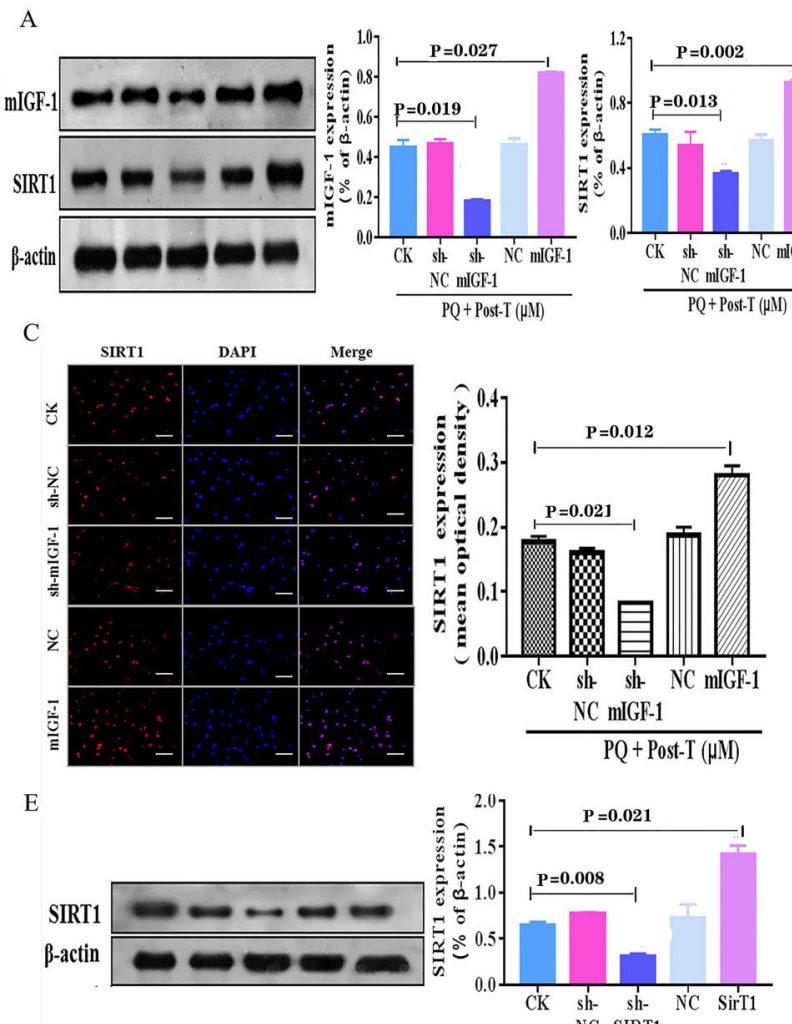

NC SIRT1

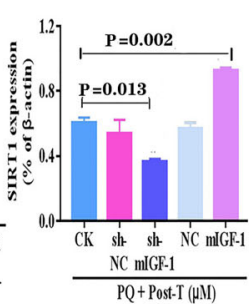

B
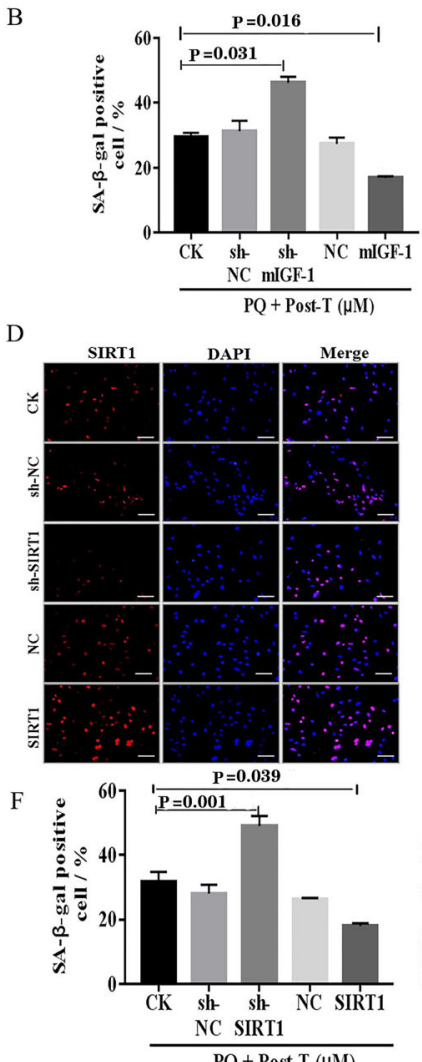

$\mathrm{PQ}+\mathrm{Post}-\mathrm{T}(\mu \mathrm{M})$
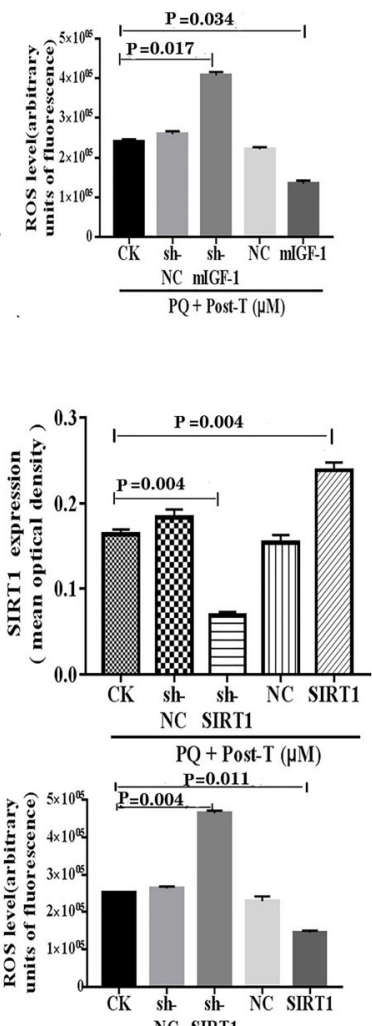

NC SIRT1

Figure 2. Testosterone $(T)$ delayed paraquat (PQ)-induced cardiomyocyte senescence via the mIGF-1/SIRT1 pathway. A, Representative western blotting and band densitometry for mIGF-1 and SIRT1 in HL-1 cardiomyocytes transfected with or without sh-mIGF-1 and mIGF-1 in the presence of PQ and T. B, Percentages of senescence-associated $\beta$-galactosidase (SA- $\beta$-gal)positive HL-1 cardiomyocytes and reactive oxygen species (ROS) production of cardiomyocytes after the same treatments as in panel A. C, Detection of SIRT1 expression in HL-1 cardiomyocytes by immunofluorescence and the statistical results after the same treatment as in panel A. Scale bar indicates $50 \mu \mathrm{m}$. D, Detection of SIRT1 expression in HL-1 cardiomyocytes by immunofluorescence and the statistical results after transfected with or without sh-SIRT1 and SIRT1 in the presence of PQ and T. Scale bar indicates $50 \mu \mathrm{m}$. E, Representative western blotting and band densitometry for SIRT1 in HL-1 cardiomyocytes after the same treatment as in panel 2D. F, Percentages of SA- $\beta$-gal-positive HL-1 cardiomyocytes and the ROS production of cardiomyocytes after the same treatments as in panel 2D. Data are reported as means \pm SE of four independent experiments (ANOVA and Dunnett's multiple comparisons test). CK: control.

and these changes were abolished by testosterone. Interestingly, the inhibition of the mIGF-1 and SIRT1 expression in PQ-induced HL-1 cardiomyocyte treated with testosterone significantly increased the expressions of ACTA1 and MHC7 but decreased the expressions of SERCA2 and MHC6 compared with testosterone alone. In brief, these results suggested that testosterone adjusted the gene expression of the contractile proteins/enzymes in senescent cardiomyocytes via mediating mIGF-1/ SIRT1 signaling pathway.

\section{Discussion}

In the present study, we confirmed that testosterone prevented $\mathrm{PQ}$-induced cardiomyocyte senescence, and further studies indicated that the protection was by regulating gene expression of the contractile proteins/ enzymes via mIGF-1/SIRT1 signaling pathway.

Although $P Q$ is widely used in agricultural practices as a quaternary nitrogen herbicide, it is extremely toxic to human beings, animals, and insects, for which there is no specific antidote (25), leading to acute lung and heart damage $(26,27)$. In particular, current studies have shown that $P Q$ treatment induces myocardial dysfunction (28-30) and senescence (31). The idea of PQ-induced cardiomyocyte senescence is further supported by data from the present study. Our results also demonstrated that $P Q$ treatment for $72 \mathrm{~h}$ induced senescence-related phenotypes in HL-1 cells, including the increase of SA- $\beta$-gal activity and ROS generation. These findings are consistent with previously reported phenotypes associated with cardiomyocyte senescence (31). 

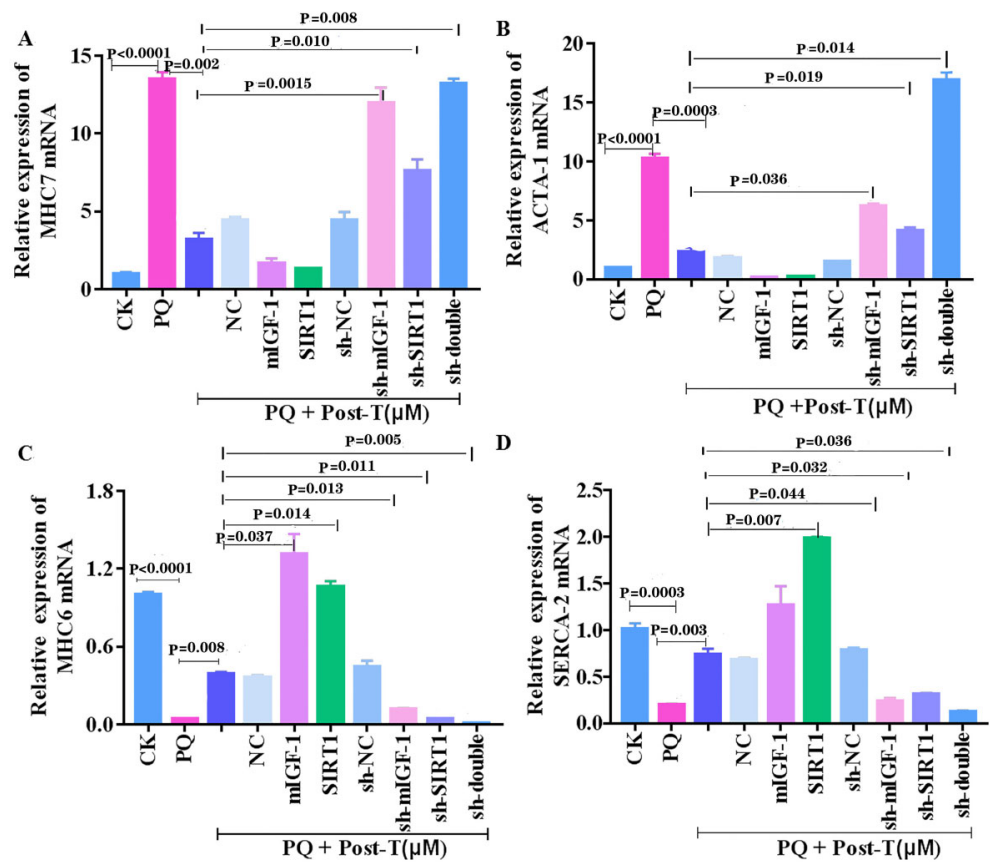

Figure 3. Testosterone $(T)$ changed the gene expression of the contractile protein/enzyme via mediating mIGF-1/SIRT1 pathway in HL-1 cardiomyocytes. The expressions of MHC7 mRNAs (A), ACTA-1 mRNAs (B), MHC6 mRNAs (C), and SERCA2 mRNAs (D) were examined by qRT-PCR. Data are reported as means \pm SE of four independent experiments (ANOVA and Dunnett's multiple comparisons test). PQ: paraquat; $\mathrm{CK}$ : control; NC: negative control.

Testosterone is the major systemic androgen and its level gradually decreased with age in men (4). Studies have shown that a low level of testosterone is closely related to the development of aging-associated cardiovascular disease, and testosterone replacement therapies play a beneficial role in male cardiovascular disease $(32,33)$. Zhang et al. $(9,34)$ found that testosterone deficiency induces cardiomyocyte senescence, and physiological testosterone therapies delay cardiomyocyte senescence via an AR-independent pathway. In addition, testosterone antagonizes doxorubicin-induced cardiomyocyte senescence by modulating telomere binding factor 2 (9).

IGF-1 and SIRT1 are novel important mediators of cell homeostasis and cardiac stress that have been connected to the lifespan regulation in multiple organisms $(23,35,36)$. Studies have revealed that circulating levels of IGF-1 significantly decline with age both in elderly human and experimental animals, and the age-related decline in circulating IGF-1 is causally implicated in the development of aging phenotype in the heart and vasculature $(12,37,38)$. Moreover, cardiac-specific overexpression of IGF-1 protects the heart from PQ-induced oxidative stress and lethality via activating SIRT1 expression (16). In addition, SIRT1 belongs to the sirtuin family of nicotinamide adenine dinucleotide (NAD)-dependent protein deacetylases, which is a crucial protective molecule against cardiovascular aging (10). SIRT1 overexpression or activation mediates anti-aging and cell-protective effects in the heart in vivo (19). Moreover, an age-mediated decrease in SIRT1 activity was observed in the heart of aged rat (39). These studies suggest that IGF-1 and SIRT1 play an important role in suppressing the aging process of organs and cells. Our results are consistent with those studies demonstrating that IGF-1 and SIRT1 levels were significantly down-regulated in $\mathrm{PQ}$-induced cardiomyocyte aging. However, it is unclear whether testosterone prevents $\mathrm{PQ}$-induced cardiomyocyte aging associated with activation of the IGF-1/SIRT1 signaling pathway. In the present study, our results indicated that testosterone intervention blocked $\mathrm{PQ}$-induced $\mathrm{HL}-1$ cell aging-related phenotypes, which were accompanied by up-regulation of IGF-1 and SIRT1 proteins. Importantly, inhibition of IGF-1 and SIRT1 significantly exacerbated senescencerelated phenotypes including increased SA- $\beta$-gal activity and ROS production in $\mathrm{PQ}$-induced $\mathrm{HL}-1$ cells with testosterone intervention, which were accompanied by the up-regulation of ACTA- 1 and MHC-7 and the downregulation of MHC- 6 and SERCA2. Therefore, it was reasonable to conclude that IGF-1 and SIRT1 were at least in part responsible for testosterone-mediated protection against the cardiomyocyte aging induced by $P Q$. Previous studies show that SIRT1 is a downstream mediator of mIGF-1 action in the heart, and mIGF-1 
protects cardiomyocytes from oxidative stress via SIRT1 activity in vitro and in vivo (16). Furthermore, cardiacrestricted mIGF-1 transgene induced systemic changes in a SIRT1-dependent manner, such as high blood pressure, leukocytosis, and an enhanced fear response (40). In the present study, our results revealed that mIGF-1 inhibition blocked SIRT1 expression/activity in PQ-induced HL-1 cells with testosterone, suggesting that testosterone activated SIRT1 via mIGF-1 to delay cardiomyocyte senescence caused by $P Q$.

There were limitations in our study: 1) the HL-1 cardiomyocyte cell line was used as the test object, and to a certain extent, it could not completely simulate the characteristics of mouse primary cardiomyocytes; 2) the effect of testosterone on aging of cardiomyocytes in our study was dose-dependent, but the dose threshold of this benefit was not proven. Therefore, an intervention with a higher dose group could provide a clearer understanding of this problem; and 3) it was not clear whether there was an internal mechanism molecule between mIGF-1 and SIRT1. Future experiments should be developed to further clarify this issue.

\section{References}

1. Cleland JG, Khand A, Clark A. The heart failure epidemic: exactly how big is it? Eur Heart $J$ 2001; 22: 623-626, doi: 10.1053/euhj.2000.2493.

2. Shirakabe A, Ikeda Y, Sciarretta S, Zablocki DK, Sadoshima J. Aging and autophagy in the heart. Circ Res 2016; 118: 1563-1576, doi: 10.1161/CIRCRESAHA.116.307474.

3. Conrad N, Judge A, Tran J, Mohseni H, Hedgecott D, Crespillo AP, et al. Temporal trends and patterns in heart failure incidence: a population-based study of 4 million individuals. Lancet 2018; 391: 572-580, doi: 10.1016/ S0140-6736(17)32520-5.

4. Araujo $A B$, O'Donnell $A B$, Brambilla DJ, Simpson WB, Longcope $C$, Matsumoto $A M$, et al. Prevalence and incidence of androgen deficiency in middle-aged and older men: estimates from the Massachusetts male aging study. $J$ Clin Endocrinol Metab 2004; 89: 5920-5926, doi: 10.1210/ jc.2003-031719.

5. Goodale T, Sadhu A, Petak S, Robbins R. Testosterone and the heart. Methodist Debakey Cardiovascular J 2017; 13: 68-72, doi: 10.14797/mdcj-13-2-68.

6. Frye CA, Kassandra E, Kanako S. Androgen administration to aged male mice increases anti-anxiety behavior and enhances cognitive performance. Neuropsychopharmacology 2008; 33: 1049-1061, doi: 10.1038/sj.npp.1301498.

7. Li ZB, Wang J, Wang JX, Chen XM, Jiang SS. Testosterone therapy improves cardiac function of male rats with right heart failure. Zhonghua Nan Ke Xue 2009; 15: 994-1000.

8. Altieri P, Barisione C, Lazzarini E, Garuti A, Bezante GP, Canepa M, et al. Testosterone antagonizes doxorubicininduced senescence of cardiomyocytes. J Am Heart Assoc 2016; 5: e002383, doi: 10.1161/JAHA.115.002383.

9. Zhang L, Wu SZ, Ruan YJ, Hong L, Xing XW, Lai WY. Testosterone therapy delays cardiomyocyte aging via an
In summary, our data demonstrated that the protective role of testosterone in $\mathrm{PQ}$-induced cardiomyocyte aging was attributed to mediating the mIGF-1/SIRT1 signaling pathway, thereby suppressing cardiac aging-associated phenotypes and modifying contractile protein/enzyme expression. Further studies are necessary to verify whether testosterone also functions in inhibition of cardiac aging via the mIGF-1/SIRT1 pathway in primary cardiomyocytes and animal models, as well as to explore the downstream signaling pathway of testosterone-regulated SIRT1 involved in anti-cardiac aging.

\section{Supplementary Material}

Click here to view [pdf].

\section{Acknowledgments}

This work was supported by research grants from Guangdong Science and Technology Planning Project of Guangdong Science and Technology Department (2014 A020212305).

androgen receptor-independent pathway. Braz J Med Biol Res 2011; 44: 1118-1124, doi: 10.1590/S0100-879X2011 007500128.

10. Luo XY, Qu SL, Tang ZH, Zhang Y, Liu MY, Peng J, et al. SIRT1 in cardiovascular aging. Clin Chim Acta 2014; 437: 106-114, doi: 10.1016/j.cca.2014.07.019.

11. Salminen A, Kaarniranta K, Kauppinen A. Crosstalk between oxidative stress and SIRT1: impact on the aging process. Int $J$ Mol Sci 2013; 14: 3834-3859, doi: 10.3390/ijms14023834.

12. Ungvari $Z$, Csiszar $A$. The emerging role of IGF-1 deficiency in cardiovascular aging: recent advances. J Gerontol A Biol Sci Med Sci 2012; 67: 599-610, doi: 10.1093/gerona/ gls072.

13. Troncoso R, Ibarra C, Vicencio JM, Jaimovic E, Lavandero S. New insights into IGF-1 signaling in the heart. Trends Endocrinol Metab 2014; 25: 128-137, doi: 10.1016/j.tem. 2013.12.002.

14. Torella D, Rota M, Nurzynska D, Musso E, Monsen A, Shiraishi I, et al. Cardiac stem cell and myocyte aging, heart failure, and insulin-like growth factor-1 overexpression. Circ Res 2004; 94: 514-524, doi: 10.1161/01.RES.0000117306. 10142.50 .

15. Davis ME, Hsieh PCH, Tomosaburo T, Song Q, Zhang SG, Kamm RD, et al. Local myocardial insulin-like growth factor 1 (IGF-1) delivery with biotinylated peptide nanofibers improves cell therapy for myocardial infarction. Proc Natl Acad Sci USA 2006; 103: 8155-8160, doi: 10.1073/pnas.0602877103.

16. Vinciguerra M, Santini MP, Martinez C, Pazienza V, Claycomb WC, Giuliani A, et al. mIGF-1/JNK1/SirT1 signaling confers protection against oxidative stress in the heart. Aging cell 2012; 11: 139-149, doi: 10.1111/j.1474-9726.2011.00766.x.

17. Cohen HY, Miller C, Bitterman KJ, Wall NR,Hekking B, Kessler B, et al. Calorie restriction promotes mammalian cell 
survival by inducing the SIRT1 deacetylase. Science 2004; 305: 390-392, doi: 10.1126/science.1099196.

18. Howitz KT, Bitterman KJ, Cohen HY, Lamming DW, Lavu S, Wood JG, et al. Small molecule activators of sirtuins extend Saccharomyces cerevisiae lifespan. Nature 2003; 425: 191-196, doi: 10.1038/nature01960.

19. Alcendor RR, Gao S, Zhai PY, Zablocki D, Holle E, Yu XZ, et al. Sirt1 regulates aging and resistance to oxidative stress in the heart. Circ Res 2007; 100: 1512-1521, doi: 10.1161/ 01.RES.0000267723.65696.4a.

20. Ota H, Akishita M, Akiyoshi T, Kahyo T, Setou M, Ogawa S, et al. Testosterone deficiency accelerates neuronal and vascular aging of SAMP8 mice: protective role of eNOS and SIRT1. Plos One 2012; 7: e29598, doi: 10.1371/journal.pone. 0029598.

21. Bruno BDJ, Blasco MA. Assessing cell and organ senescence biomarkers. Circ Res 2012; 111: 97-109, doi: 10.1161/CIRCRESAHA.111.247866.

22. Davalli P, Mitic T, Caporali A, Lauriola A, D’Arca D. ROS, cell senescence, and novel molecular mechanisms in aging and age-related diseases. Oxid Med Cell Longev 2016: 3565127 , doi: 10.1155/2016/3565127.

23. Guarente L. Franklin H. Epstein lecture: sirtuins, aging, and medicine. N Engl J Med 2011; 364: 2235-2244, doi: 10.1056/NEJMra1100831.

24. Ge W, Zhang YM, Han XF, Ren J. Cardiac-specific overexpression of catalase attenuates paraquat-induced myocardial geometric and contractile alteration: role of ER stress. Free Radical Biol Med 2010; 49: 2068-2077, doi: 10.1016/j.freeradbiomed.2010.10.686.

25. Silva R, Carmo H, Vilas-Boas V, Barbosa DJ, Monteiro M, Pinho PG, et al. Several transport systems contribute to the intestinal uptake of Paraquat, modulating its cytotoxic effects. Toxicol Lett 2015; 232: 271-283, doi: 10.1016/ j.toxlet.2014.10.015.

26. Noguchi N, Tanaka E, Yamamoto H, Misawa S. Initial accumulation of paraquat in the heart leading to acute death. Nihon Hōigaku Zasshi 1990; 44: 6-11.

27. Yan BL, Chen F, Xu LJ, Xing JH, Wang XF. HMGB1-TLR4IL23-IL17A axis promotes paraquat-induced acute lung injury by mediating neutrophil infiltration in mice. Sci Rep 2017; 7: 597, doi: 10.1038/s41598-017-00721-8.

28. Jiang W, Mitra R, Lin CC, Wang Q, Cheng FX, Zhao ZM. Systematic dissection of dysregulated transcription factormiRNA feed-forward loops across tumor types. Brief Bioinform 2016; 17: 996-1008, doi: 10.1093/bib/bbv107.

29. Wang SY, Zhu XL, Xiong LZ, Ren J. Ablation of Akt2 prevents paraquat-induced myocardial mitochondrial injury and contractile dysfunction: role of Nrf2. Toxicol Lett 2017; 269: 1-14, doi: 10.1016/j.toxlet.2017.01.009.

30. Zhang LP, Feng QM, Wang T. Necrostatin-1 protects against paraquat-induced cardiac contractile dysfunction via RIP1RIP3-MLKL-dependent necroptosis pathway. Cardiovas Toxicol 2018; 18: 346-355, doi: 10.1007/s12012-0179441-z.

31. Chang ZS, Xia JB, Wu HY, Peng WT, Jian FQ, Li J, et al. Forkhead box $\mathrm{O} 3$ protects the heart against paraquatinduced aging-associated phenotypes by upregulating the expression of antioxidant enzymes. Aging Cell 2019; 18: e12990, doi: 10.1111/acel.12990.

32. Santos MR, Sayegh ALC, Groehs RVR, Fonseca G, Trombetta IC, Barretto ACP, et al. Testosterone deficiency increases hospital readmission and mortality rates in male patients with heart failure. Arq Bras De Cardiol 2015; 105: 256-264, doi: 10.5935/abc.20150078.

33. Yeap BB, Flicker L. Hormones and cardiovascular disease in older men. J Am Med Dir Assoc 2014; 15: 326-333, doi: 10.1016/j.jamda.2013.12.004.

34. Zhang L, Lei D, Zhu GP, Hong L, Wu SZ. Physiological testosterone retards cardiomyocyte aging in $\mathrm{Tfm}$ mice via androgen receptor-independent pathway. Chin Med Sci J 2013; 28: 88-94, doi: 10.1016/S1001-9294(13)60028-0.

35. Fontana L, Vinciguerra M, Longo VD. Growth factors, nutrient signaling, and cardiovascular aging. Circ Res 2012; 110: 1139-1150, doi: 10.1161/CIRCRESAHA.111. 246470.

36. Lavu S, Boss O, Elliott PJ, Lambert PD. Sirtuins-novel therapeutic targets to treat age-associated diseases. Nat Rev Drug Discov 2008; 7: 841-853, doi: 10.1038/nrd2665.

37. D'Costa AP, Ingram RL, Lenham JE, Sonntag WE. The regulation and mechanisms of action of growth hormone and insulin-like growth factor 1 during normal ageing. J Reprod Fertil Suppl 1993; 46: 87-98.

38. Khan AS, Sane DC, Thomas W, Sonntag WE. Growth hormone, insulin-like growth factor- 1 and the aging cardiovascular system. Cardiovas Res 2002; 54: 25-35, doi: 10.1016/S0008-6363(01)00533-8.

39. Ferrara N, Rinaldi B, Corbi G, Conti V, Stiuso P, Boccuti S, et al. Exercise training promotes SIRT1 activity in aged rats. Rejuvenation Res 2008; 11: 139-150, doi: 10.1089/rej. 2007.0576.

40. Bolasco G, Calogero R, Carrara M, Banchaabouchi MA, Bilbao D, Mazzoccoli G, et al. Cardioprotective mIGF-1/ SIRT1 signaling induces hypertension, leukocytosis and fear response in mice. Aging 2012; 4: 402-416, doi: 10.18632/ aging. 100464 . 